\title{
Multi-product multi-vehicle inventory routing problem with vehicle compatibility and site dependency: A case study in the restaurant chain industry
}

\author{
Shunichi Ohmori ${ }^{a^{*}}$ and Kazuho Yoshimoto ${ }^{a}$
}

${ }^{a}$ Waseda University, Okubo 3-4-1, Shinjuku, Tokyo 169-8555, Japan

\section{A B S T R A C T}

\begin{abstract}
We study an inventory routing problem (IRP) for the restaurant chain. We proposed a model a multi-product multi-vehicle IRP (MMIRP) with multi-compatibility and site-dependency (MMIRP-MCSD). The problem was formulated as a mixed integer programming (MIP). This model is difficult to solve because it is a problem that integrates MMIRP, a multi-compartment vehicle routing problem (MCVRP), and a site dependent VRP (SDVRP), each of which is difficult even by itself. Therefore, in this study, we proposed three-stage Math Heuristics based on the cluster-first and route-second method. In the numerical experiment, verification was performed using actual data, and knowledge on the decision making of the optimum vehicle type was obtained.
\end{abstract}

\section{Introduction}

Logistics is a social infrastructure that supports industrial competitiveness and wealthy lives of people, and thus must never be interrupted. However, economies with decreasing and aging populations make it difficult to maintain the infrastructure in many countries. For example, with cases in Japan, the population has started to decline since 2011, and is expected to continue to decline. The working-age population is also expected to decline in the future. The aging of truck drivers and the shortage of labor are already becoming serious, and there is a possibility of substantial luck of the workforce in the future. Further, in an area where demand is small, such as a depopulated area, a decrease in the amount of cargo makes delivery to the area difficult. On the other hand, the number of freight movements is rapidly increasing along with the growth of e-commerce and omni-channel, and there is a concern that transportation efficiency will decrease due to smaller and more frequent transportations. In addition, the needs for logistics have also changed significantly, such as a samedelivery option. In the future, as the needs for logistics become more complex, the logistics crisis can become the bottleneck of the economy. The improvement efforts of individual companies are already reaching their limits, and in order to dramatically improve delivery efficiency, it is necessary to work on optimization of the entire supply chain based on cooperation between organizations.

The Inventory Routing Problem (IRP) is one of the studies on improving delivery efficiency based on such cooperation. IRP is a problem in which a logistics company and a customer work together to make a decision that integrates the customer's inventory management and the logistics company's delivery plan. Specifically, three decisions are made: (1) when to deliver, (2) how much to deliver, and (3) by what route. When each customer independently places a delivery request to a logistics company, the delivery timing is randomly dispersed, which forces individualized delivery, leading to frequent small-quantity delivery and reducing delivery efficiency. Therefore, it can be expected that high delivery efficiency will be achieved by coordinating the delivery timing with the customer and the logistics company. IRP has a history of more than 30 years, originating from the work of Bell et al. (1983). A survey paper is Coelho et al. (2013). The various variations of the model include a combination of features as shown in Table 1.

* Corresponding author

E-mail address: ohmori0406@aoni.waseda.jp (S. Ohmori)

(C) 2021 by the authors; licensee Growing Science. doi: $10.5267 /$ j.uscm.2021.2.007 
Table 1

Structural Variance of IRP (Coelho et al. 2013) and assumptions in this research (in bold)

\begin{tabular}{ll}
\hline \multicolumn{1}{c}{ Characteristics } & \multicolumn{1}{c}{ Options } \\
\hline Time horizon & Finite / Infinite \\
Structure & One to Many / Many to One / Many to Many \\
Routing & Direct / Multiple / Continuous \\
Inventory Policy & Maximum Level / Order-up-to / Optimal \\
Inventory Decisions & Lost Sales / Back-order / Nonnegative \\
Vehicle Type & Homogeneous / Heterogeneous \\
Number of Vehicles & Single / Multiple / Unconstrained \\
\hline
\end{tabular}

Table 2

Vehicle type to consider

\begin{tabular}{ccccc}
\hline$r$ & Vehicle size & Temperature zone & Dry & Chilled \\
\hline 1 & Medium & Three-temperature-zone & $\checkmark$ & $\checkmark$ \\
2 & Medium & Room-temperature & $\checkmark$ & $\checkmark$ \\
3 & Large & Three-temperature-zone & $\checkmark$ & $\checkmark$ \\
4 & Large & Room-temperature & $\checkmark$ & $\checkmark$ \\
\hline
\end{tabular}

In this study, we conduct the following case studies based on interviews with a leading restaurant chain, which is expanding its business all over the world. There is one Regional DCs, from which it delivers to multiple stores. Each store handles items in three temperature zones: dry (room temperature), chilled, and frozen. Examples of frozen items include meat, examples of refrigerated items include vegetables, and examples of room dry items include packaging materials. Each store receives multiple deliveries during a week. Dry items, chilled items, and dry items must be replenished so that they will not run out of stock. The same pattern is repeated every week. To determine the delivery pattern, the demand forecast for each item at each store on each day of the week is used. The demand for each item is aggregated into standard packaging units for each temperature zone. The demand of each store is different for each day of the week. In addition, although the actual delivery amount is different from the predicted value, if it cannot be carried by the delivery pattern obtained in this study, it is delivered by using another vehicle.

In this study, we consider the decision to select the most appropriate one from among the four vehicle types shown in Table 1 based on interviews with restaurant chains. This raises two new operational challenges. The first challenge is the consideration of multiple temperature zones. Vehicle type are three-temperature-zone vehicles and can deliver items in all temperature zones. On the other hand, vehicle type is room temperature vehicles and can only deliver dry items at room temperature. Generally, if the car size is the same, the room-temperature car is cheaper. However, since room-temperature vehicles cannot deliver chilled and frozen items, it is necessary for three temperature zone vehicles to deliver frozen and chilled items to the same store at different timings. Therefore, it is necessary to consider the trade-off: should we use three temperature zone vehicles to deliver items in each temperature zone to the same store at once, or should we use room temperature vehicles and deliver to the same store at different timings for each temperature zone? Fig. 1 shows an example illustrating the inventory routing decision with and without a room temperature vehicle. Figure 1 (a) shows an example of delivery using only three temperature zones. Fig. 1 (b) is an example when both the three temperature zones and the room temperature car are used. At $t=1$, only normal temperature items are delivered using a normal temperature vehicle. On the other hand, at $\mathrm{t}=2$ and 3 , only frozen and refrigerated products are delivered using a three-temperature zone vehicle.

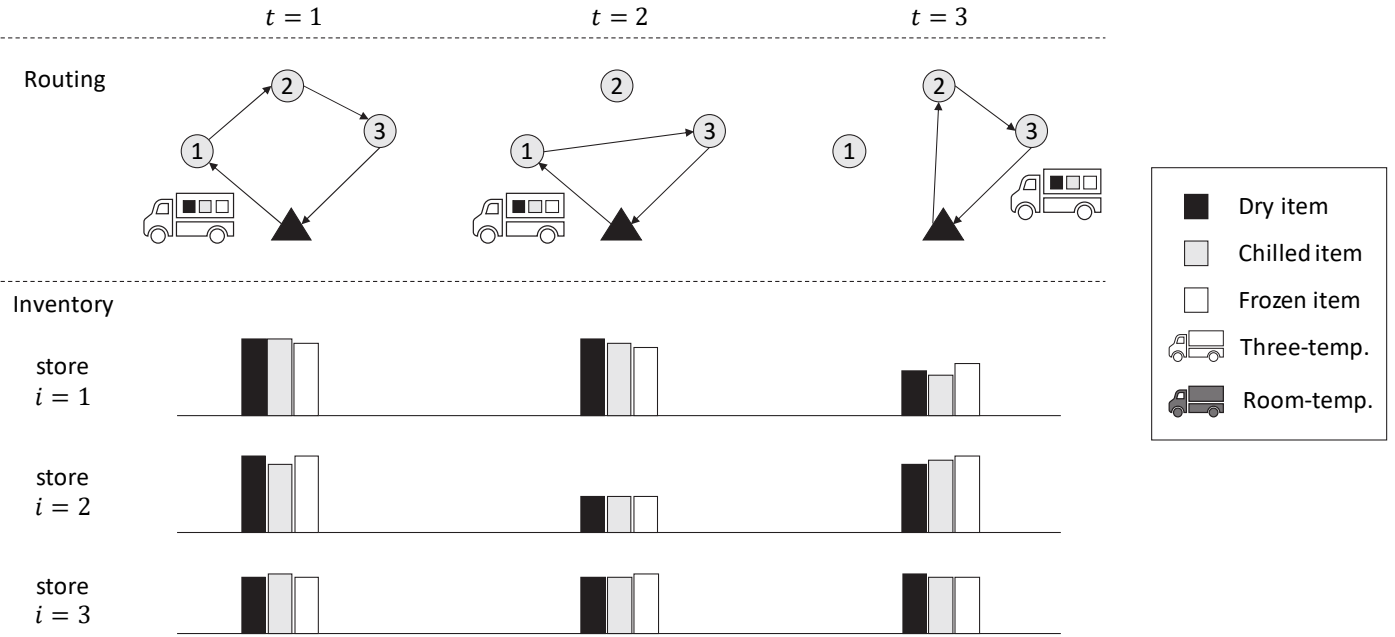

(a) A case where only three-temperature vehicles are used 


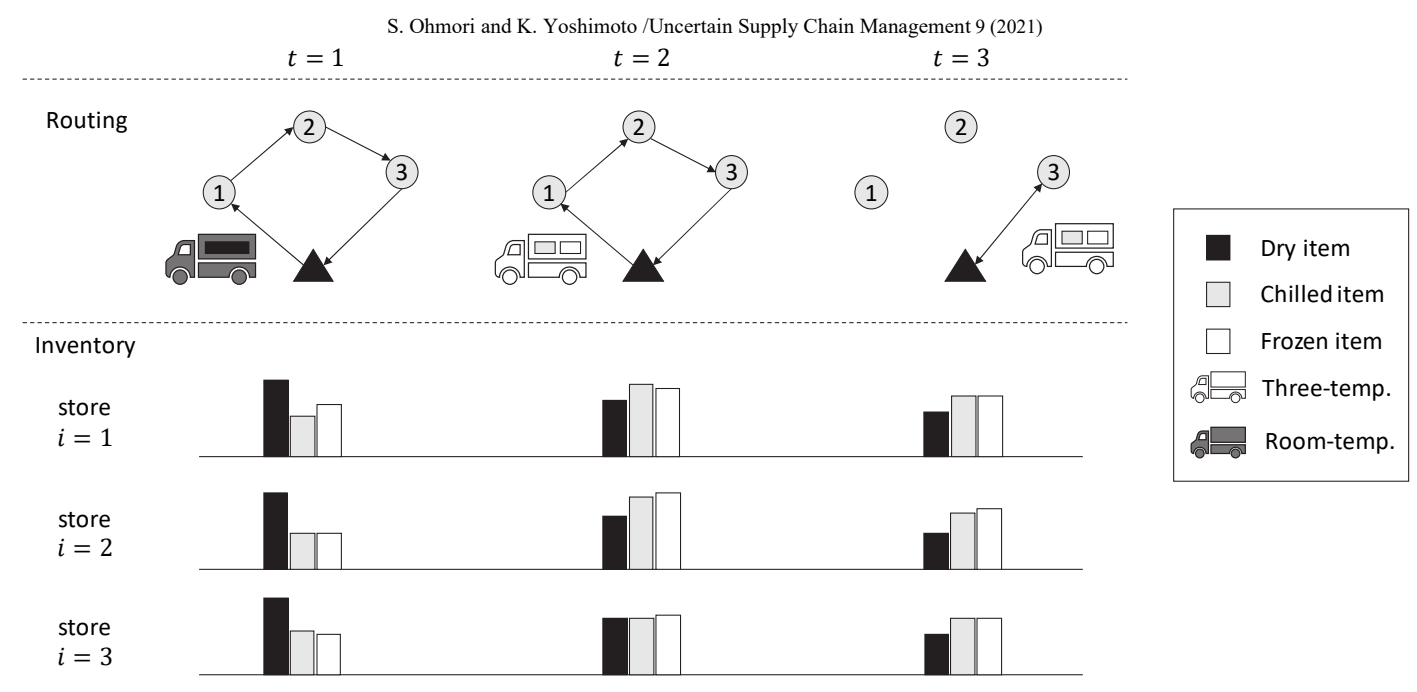

(b) A case where three-temperature vehicles and a room-temperature are used

Fig. 1. An example illustrating the inventory routing decisions with different temperature-zone vehicles
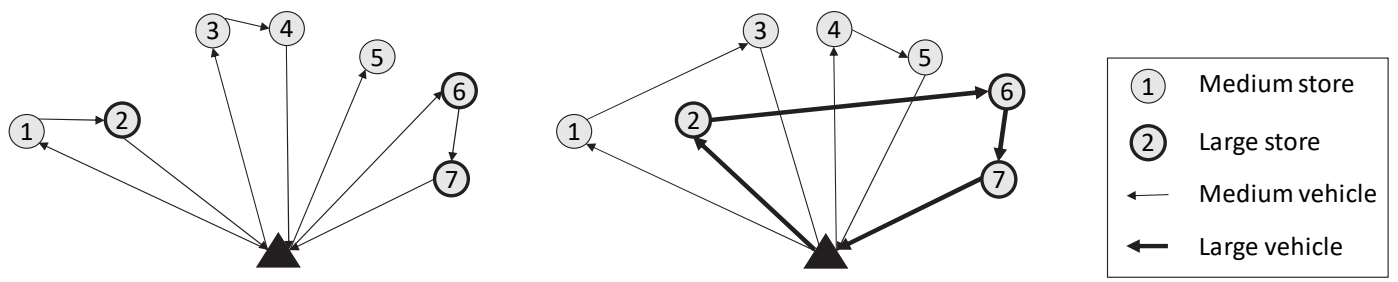

(b)A case with medium and large truck

(a)A case with medium truck

Fig. 2. An example illustrating the routing decision with different size vehicles

The second challenge is vehicle type restrictions. As shown in Table 2, this research deals with two types of vehicles, large and medium. In general, when compared on a per-weight basis, large vehicles are less costly. On the other hand, there are stores that large vehicles cannot enter or park in, mainly in stores in the downtown area. Therefore, it is necessary to consider the trade-off between cost and delivery availability. Fig. 2 shows an example of a delivery plan with and without a large vehicle. In this example, Fig. 2 (a) uses only medium vehicles for delivery. Although the distance between each route is short, the number of stores delivering within one route is small due to the constraint on loading capacity. On the other hand, Fig. 2 (b) uses both small and large vehicles for delivery. By using a large vehicle, more stores can be delivered by one route, but the delivery distance is longer.

\section{Literature review}

This section discusses related studies. Section 2.1 summarizes research on IRP. Section 2.2 summarizes the Multi-Product Multi-Vehicle IRP. Section 2.3 discusses the contribution of this research.

\section{$2.1 \operatorname{IRP}$}

IRP has a history of more than 30 years of research, and various variants of models have been proposed. In addition, many application examples have been published, such as chemical products (Dauzere-Peres et al., 2007), oil and gas (Song \&Furman 2013) and other applications in the maritime industry, food (Gaur and Fisher 2004), cement (Christiansen et al. 2011), Gasoline (Popovic et al. 2012), blood (Hemmelmayr et al., 2009), organs (Oppen et al., 2010) and waste oil (Aksen et al. 2012). IRP is a difficult problem that integrates inventory planning and delivery planning, and much research has been done on how to solve it. The exact solution is Branch-and-cut (Archetti et al. 2007, Coelho and Laporte 2013, Desaulniers 2016, Avella et al. 2018) and Dynamic programming (Kleywegt et al. 2004) have been proposed. Approximate solutions include Tabu search (Cousineau-Ouimet, 2002), variable neighborhood search (Liu \& Lee, 2011; Liu \& Chen 2012, Mjirda et al., 2014), genetic algorithms (Park et al., 2016) has been applied. In recent years, math-heuristics that combine mathematical programming and metaheuristics have been proposed. Guerrero et al. (2013) studied the Location Inventory Routing Problem, and in the first stage, the location, inventory, and supply depot for each customer were determined by MIP. We proposed a method using Local Search in two steps. Cordeau et al. (2015) proposed the problem of determining 
delivery timing and delivery volume in the first stage and determining the delivery route in the second stage. The first stage proposes a method of resolving the problem using Lagrange relaxation. Su et al. (2020) proposed mass heuristics using local search for route search, MIP for delivery timing, and LP for delivery volume determination.

\subsection{MMIRP}

The IRPs that handle multiple items are as follows. Speranza and Ukovich (1994) studied the multi-product IRP in a single customer with a fixed delivery frequency. Bertazzi et al. (1997) dealt with the problem of supplying multiple types of fuel and extended it to the problem of multiple customers. Morin et al. (2011) dealt with the issue of shipping from multiple suppliers to a single customer. Mjirda et al. (2012) addressed the issue of shipping from multiple suppliers to multiple customers. Ronen (2002) applied multi-product IRP to the maritime shipping industry. Mirzapour and Rekik (2014), Treitl et al. (2014), Alkawaleet et al. (2014) proposed a model that considers carbon dioxide emissions. Rahimi et al. (2017) proposed a multi-objective optimization model that takes customer satisfaction into account. Lmariouh et al. (2017) studied models that supply bottled water of different sizes. Benoist et al. (2011), Coelho and Laporte (2013), Cordeau et al. (2015), Ercan and Cinar (2017) studied MMIRP with multiple vehicles. Branch-and-Cut of Laporte (2013) has been proposed as an exact solution, but even in the largest instance, 5 items, 5 units per model, 3 periods Since there were 30 customers, heuristics would need to be applied in order to apply to larger issues.

On the other hand, Heterogeneous VRP has been studied for more than 30 years for delivery plans using multiple vehicle types. Baldacci (2008) and Koc (2016) are detailed as survey papers. In particular, those closely related to this study are the Multi-Compartment VRP (Wang et al., 2014) and Site-Dependent VRP (Chao et al., 1999; Nag et al., 1988). Considering these requirements, it is difficult to solve the delivery plan alone, and as far as we know, there are no studies considering it in combination with IRP.

\subsection{Contribution of the work}

The contributions of this research are as follows:

1. The first novelty is that we proposed a model that incorporates vehicle compatibility and site dependency into the MMIRP, which have not been considered previously in the MMIRP, based on the case of a restaurant chain with multiple temperature zones and multiple vehicle types of delivery. When considering both, it is necessary to clarify when, which items, which vehicle type, which store, and how much of the item is transported, but the previous research has not clarified these points.

2. The second novelty is that we proposed a solution to the above problem. In this study, we propose a three-stage heuristic that applies the idea of cluster-first and root-post method. In the first stage, a model called Multi-Product Multi-Vehicle Inventory Problem (MMIP) is proposed and the delivery destinations are clustered by delivery day and vehicle type. In the second stage, we solve the Vehicle Location Problem to cluster the delivery destinations by delivery vehicles. In the third step, by solving the Travelling Salesman Problem (TSP) for each delivery vehicle, the delivery routes are determined. In this way, the original problem, which is difficult to solve, is decomposed into solvable partial decisions, making it possible to apply the method to problems of a scale that could not be solved in the past.

3. The third novelty of this research is that it was verified using actual data from a restaurant chain. Although IRP has been applied to various industries, this is the first time it has been applied to the restaurant industry. In particular, it is an unprecedented achievement that we have clarified the ideal of an inventory delivery plan that combines multiple vehicle types and its effectiveness in a numerical experiment.

\section{The proposed model}

Let $G=\{V, A\}$ denote a graph, $V=\{i \mid i=0, \cdots, n\}$ denote a set of nodes, and $A=\{(i, j) \mid i, j \in N\}$ denote a set of arcs. Node 0 is the warehouse and node and $1, \cdots, n$ are the store nodes. Let $V^{\prime}=V \backslash\{0\}$ denote a set of store nodes. We aggregate items by the temperature zone. Let $S=\{s \mid s=1, \cdots, 3\}$ denote a set of items grouped by the temperature zone, $s=1$ is the dry (or room-temperature) items, $s=2$ is the chilled items, and $s=3$ is the frozen items. Let $T=\{1, \cdots, 7\}$ denote a set of periods and corresponds to the day of the week as Monday $(t=1)$ to Sunday $(t=7)$, respectively. Let $d_{i t s}$ denote the demand of item $s$ in period $t$ of the store $i$. Weekly demand is stable, and we can expect $d_{i t s}=d_{i(t+7) s}$, that is, to repeat the same cycle of demand. Let $I_{i t s}$ denote the end-of-period inventory amount of item $s$ in period $t$ at the store $i$, and no shortages or backlogs are allowed. Each store $i \in V^{\prime}$ has a storage capacity of $W_{i s}$ of item $s$.

Let $R=\{r \mid r=1, \cdots, 4\}$ denote a set of the vehicle types. Let $g_{s r}$ denote a binary constant indicating 1 if vehicle type $r$ can load item $s$, and 0 otherwise. $g_{1 r}=g_{2 r}=g_{3 r}=1$ for the three-temperature vehicle, $g_{1 r}=1, g_{2 r}=g_{3 r}=0$ for the room-temperature vehicle. Also, let $h_{\text {ir }}$ denote a binary constant indicating 1 if the vehicle type $r$ can load the item $s$, and 0 otherwise. Let $Q_{r}$ denote the loading limit for vehicle type $r$. Let $c_{r}^{F}$ denote the fixed cost of vehicle type $r$, and $c_{i j r}^{V}$ 
denote the variable cost of moving arc $(i, j)$ with vehicle type $r$. Let $K=\{1, \cdots, m\}$ denote a set of vehicles. Also, $K_{r}=$ $\left\{\sum_{r^{\prime}=1}^{r-1} m_{r^{\prime}}+1, \cdots, \sum_{r^{\prime}=1}^{r} m_{r^{\prime}}\right\} \subseteq K$ is a set of vehicles of vehicle type $r$, and $m_{r}$ is the number of vehicles of vehicle type $r$. For each vehicle $k \in K_{r}$, loading limit $Q_{k}$, fixed $\operatorname{cost} c_{k}^{F}$, variable $\operatorname{cost} c_{i j k}^{V}$, item deliverability $g_{k s}$, sitedependency $h_{k i}$ inherits the parameters of vehicle type $r$.

Let $x_{i j t k}$ denote a binary variable that takes 1 if vehicle $k$ travels arc $(i, j)$ in period $t$, otherwise 0 . Let $y_{i t k}$ denote a binary variable that takes 1 if vehicle $k$ visits the store $i$ in period $t$, otherwise 0 . Also, let $q_{i t s k}$ denote the delivery amount of item $s$ to store $i$ by vehicle $k$ in period $t$.

\section{Set}

$V=\{i \mid i=0, \cdots, n\}:$ A set of nodes

$T=\{t \mid t=1, \cdots, 7\}:$ A set of periods

$S=\{s \mid s=1, \cdots, 3\}:$ A set of products

$K=\{k \mid k=1, \cdots, m\}:$ A set of vehicles

\section{Parameters}

$d_{i t s}$ : Demand of item $s$ in period $t$ of the store $i$.

$W_{i s}$ : Storage capacity of item $s$ at store $i$

$Q_{k}$ : loading limit of vehicle $k$

$c_{k}^{F}$ : Fixed cost of vehicle $k$

$c_{i j k}^{V}$ : Variable cost for vehicle $k$ to travel arc $(i, j)$.

$g_{s k}$ : Binary constant indicating whether item $s$ can be loaded by vehicle $k$.

$h_{i k}$ : Binary constant indicating whether store $i$ can be visited by vehicle $k$.

\section{Decision variables}

$x_{i j t k}$ : Binary variable indicating whether vehicle $k$ travels arc $(i, j)$ in period $t$.

$q_{i t s k}$ : Delivery amount of item $s$ to store $i$ by vehicle $k$ in period $t$

$I_{i t s}$ : End-of-period inventory amount of item $s$ in period $t$ at the store $i$

$y_{i t k}$ : Binary variable indicating whether vehicle $k$ visits the store $i$ in period $t$

$\min . \quad \sum_{j \in V} \sum_{t \in T} \sum_{k \in K} c_{k}^{F} x_{0 j t k}+\sum_{i \in V} \sum_{j \in V} \sum_{t \in T} \sum_{k \in K} c_{i j}^{V} x_{i j t k}$

subject to

$I_{i t s}=I_{i(t-1) s}+\sum_{k \in K} g_{s k} h_{i k} q_{i t s k}-d_{i t s} \quad \forall i \in V^{\prime}, \forall t \in T, \forall s \in S$

$I_{i t s} \leq W_{i s}, \quad \forall i \in V^{\prime}, \forall s \in S$

$q_{i t s k} \leq W_{i s} y_{i t k}, \quad \forall i \in V^{\prime}, \forall t \in T, \forall s \in S, \forall k \in K$

$\sum_{i \in V^{\prime}} q_{i t s k} \leq Q_{k} y_{0 t k}, \quad \forall s \in S, \forall k \in K$

$\sum_{j \in V} x_{i j t k}=\sum_{i \in V} x_{j i t k}=y_{i t k}, \quad \forall i \in V, \forall t \in T, \forall k \in K$

$\sum_{i, j \in X} x_{i j t k} \leq|X|-1 \quad \forall X \subseteq\{2, \cdots, n\}, \forall k \in K$

$I_{i t s} \geq 0, \quad \forall i \in V^{\prime}, \forall s \in S$

$q_{i t s k} \geq 0, \quad \forall i \in V^{\prime}, \forall s \in S, \forall k \in K$

$x_{i j t k} \in\{0,1\}, \quad \forall(i, j) \in A, \forall s \in S, \forall k \in K$

$y_{i t k} \in\{0,1\}, \quad \forall i \in N, \forall k \in K$

The objective function (1a) is the minimization of shipping costs, which consists of fixed and variable costs. The constraint (1b) indicates the inventory update formula. It is assumed that the weekly inventory delivery pattern is the same, and $I_{i 0 s}=$ $I_{i 7 s}$. The constraint (1c) indicates the upper limit of the store's storage capacity. The constraint (1d) indicates that the delivery volume will be 0 on days when delivery is not performed. The constraint (1e) indicates the load limit for each vehicle. The constraint (1f) indicates the integrity of the route. The constraint (1g) indicates the removal of the sub tour. The constraint (1h) (1i) indicates the non-negative condition of $I_{i t s}, q_{i t s k}$. The constraint $(1 \mathrm{j})(1 \mathrm{k})$ indicates the binary condition of $x_{i j t k}, y_{i t k}$. 


\section{The proposed method}

The problem (1) is a difficult problem that integrates inventory planning and delivery planning. Therefore, in this study, we propose three-stage Math Heuristics based on the cluster-first and route-second method. In the first stage, delivery day of the week, delivery vehicle type, and delivery amount for each store are determined so that the fixed vehicle cost is minimized while satisfying the upper and lower limit constraints of inventory. In this study, this is called the Multi-product MultiVehicle Inventory Problem (MMIP). From the output of MMIP, delivery clusters can be created for each delivery day and delivery vehicle type. Fig. 3 shows an example of clustering obtained from the first stage MMIP. In this example, there are 10 stores, with stores $1-5$ being large stores and stores $6-10$ being small stores. At $t=1$, all stores are assigned to mediumsized vehicles in the three temperature zones. At $t=2$, the normal temperature of stores 1-4 is assigned to normal temperature large vehicles. The normal temperature of stores 5-6 is assigned to normal temperature medium-sized vehicles. In addition, frozen and refrigerated products in stores 4-8 are assigned to medium-sized vehicles in the three temperature zones. Other stores / items will not be delivered. At $\mathrm{t}=3$, all items in stores 1-4 are assigned to large vehicles in the three temperature zones. In addition, all temperature zones in store 7 and frozen / refrigerated products in stores 8-10 are assigned to mediumsized vehicles in the three temperature zones. Room temperature products in stores 8-10 are assigned to normal temperature medium-sized vehicles. Other stores / items will not be delivered.

For these clusters, the optimum vehicle allocation and delivery route can be obtained by solving the VRP (Vehicle Routing Problem). Since the VRP needs to be solved for each of the clusters, the computation time could be longer. Therefore, we use Locational-based Heuristics, which is one of the effective heuristics that can solve VRP fast, and further decompose the problem into the store clustering (second stage) and routing (third stage) for each delivery vehicle. In the second stage, the vehicle location problem (VIP) is solved to determine the delivery vehicle for each store so that the approximate delivery variable cost is minimized. The output of this VLP creates a delivery destination cluster for each delivery vehicle. In the third stage, the Traveling Salesman Problem (TSP) is solved to determine the delivery route.

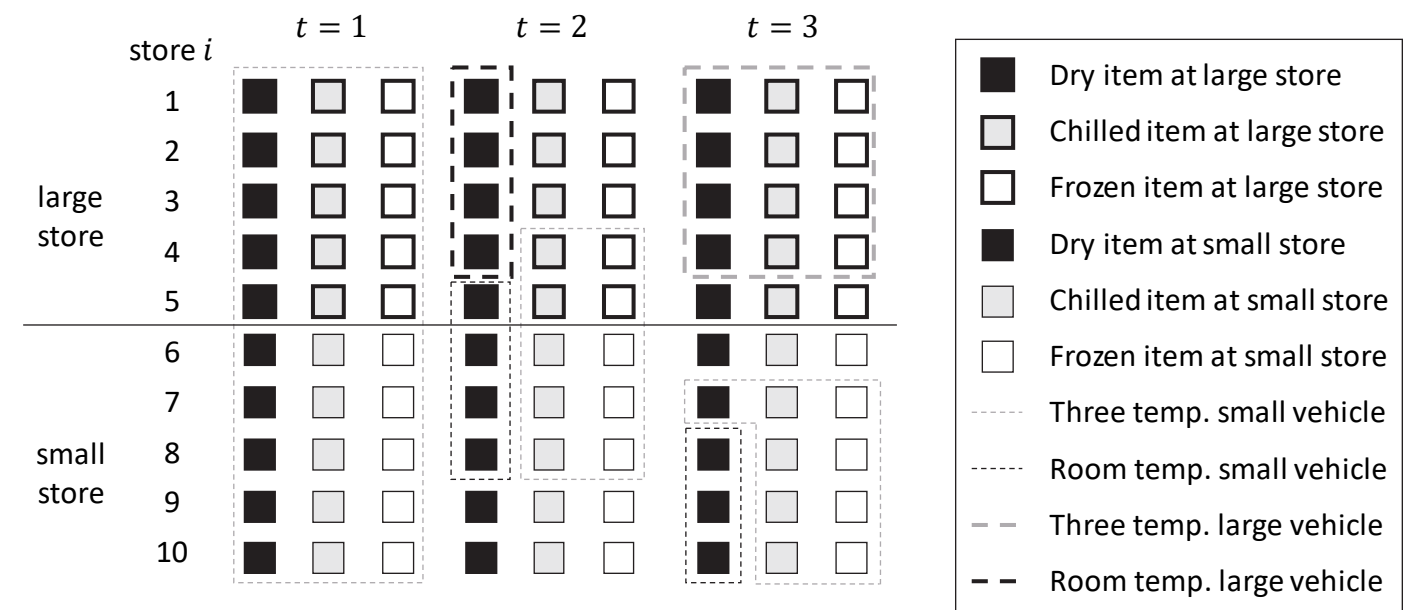

Fig. 3. An example illustrating the clustering obtained from the MMIP at $1^{\text {st }}$ stage

\subsection{First stage: Multi-Product Multi-Vehicle Inventory Problem}

In the first stage, the delivery day, the delivery vehicle type, and the delivery amount are determined for each store to cluster the stores by the delivery day and the delivery vehicle type. Let $\tilde{y}_{i t r}$ denote a binary variable that takes 1 if the store $i$ is visited in period $t$ by the vehicle of type $r$, otherwise 0 . Let $\tilde{q}_{i t s r}$ denote the delivery amount of item $s$ to store $i$ by vehicle of type $r$ in period $t$. Let $\tilde{v}_{t r}$ be the number of vehicles of type $r$ in period $t$.

$$
\begin{aligned}
& \min _{\text {subject to }} \sum_{t \in T} \sum_{r \in R} c_{r}^{F} \tilde{v}_{t r} \\
& I_{i t s}=I_{i(t-1) s}+\sum_{r \in R} g_{r s} h_{i r} \tilde{q}_{i t s r}-d_{i t s}, \quad \forall i \in V^{\prime}, \forall t \in T, \forall s \in S \\
& I_{i t s} \leq W_{i s}, \quad \forall i \in V^{\prime}, \forall t \in T, \forall s \in S \\
& \tilde{q}_{i t s r} \leq W_{i s} \tilde{y}_{i t s r}, \quad \forall i \in V, t \in T, s \in S, r \in R \\
& \sum_{i \in V} \sum_{s \in S} \tilde{q}_{i t s r} \leq Q_{r} \tilde{v}_{t r}, \quad \forall t \in T, \forall r \in R \\
& \sum_{s \in S} \tilde{q}_{i t s r} \leq Q_{r}, \quad \forall i \in V^{\prime}, \forall t \in T, \forall r \in R
\end{aligned}
$$


$I_{i t s} \geq 0, \quad \forall i \in V^{\prime}, \forall s \in S$

$\tilde{q}_{i t s r} \geq 0, \quad \forall i \in N, \forall t \in T, \forall s \in S, \forall r \in R$

$\tilde{v}_{t r} \in\left\{0,1, \cdots, m_{r}\right\}, \quad \forall t \in T, \forall r \in R$

$\tilde{y}_{i t s r} \in\{0,1\}, \quad \forall i \in N, \forall t \in T, \forall s \in S, \forall r \in R$

The objective function ( $2 a$ ) shows the sum of fixed costs. The constraint ( $2 b$ ) indicates the inventory update formula. The constraint (2c) indicates the upper limit of the store's storage capacity. The constraint $(2 \mathrm{~d})$ indicates that the delivery volume be 0 on days when delivery is not performed. The constraint (2e) indicates that the total load of each vehicle type does not exceed the total load limit of each vehicle type. The constraint (2f) indicates that the amount of one delivery to the store by each vehicle type does not exceed the load limit of each vehicle type. The constraint $(2 \mathrm{~g})-(2 \mathrm{~h})$ indicate the non-negativity condition of $I_{i t s}, \tilde{q}_{i t s k}$. The constraint (2i) indicates an integer condition of $\tilde{v}_{t r}$. The constraint (2j) indicates the binary condition of $\tilde{y}_{i t s r}$.

By the first stage clustering, it can be decomposed into clusters $C_{t r}$. Let $V_{t r}^{\prime}$ denote the set of stores contained in the cluster $C_{t r}$, and let $V_{t r}=V_{t r}^{\prime} \cup\{0\} . X_{i j t k}$ can be obtained by solving $\left(V R P_{t r}\right)$ for each cluster $C_{t r}$ shown in the Eq. (3).

$$
\begin{aligned}
& \min . \quad \sum_{i \in V_{t r}} \sum_{j \in V_{t r}, i<j} \sum_{k \in K_{r}} c_{i j k}^{V} x_{i j t k} \\
& \sum_{i \in V_{t r}^{\prime}}^{\text {subject to }} \sum_{S \in S} \tilde{q}_{i t s r} \leq Q_{k} y_{0 t k}, \quad \forall k \in K_{r} \\
& \sum_{j \in V_{t r}} x_{i j t k}=\sum_{i \in V_{t r}} x_{j i t k}=y_{i t k}, \quad \forall k \in K_{r} \\
& \sum_{i, j \in X} x_{i j t k} \leq|X|-1 \quad \forall X \subseteq\{2, \cdots, n\}, \forall k \in K_{r} \\
& x_{i j t k} \in\{0,1\}, \quad \forall i, j \in V_{t r}, \forall k \in K_{r} \\
& y_{i t k} \in\{0,1\}, \quad \forall i \in V_{t r}, \forall k \in K_{r}
\end{aligned}
$$

The objective function ( $3 a$ ) indicates the minimization of variable costs. The constraint ( $3 b)$ indicates the load limit. The constraint (3c) indicates the flow conservation of the route. The constraint (3d) indicates the removal of the sub tour. The constraint (3e)(3f) indicates the binary condition of $\tilde{x}_{i j t k}, \tilde{y}_{i t k}$.

In this study, it takes a lot of calculation time to solve VRP (3) for each cluster. Therefore, we apply Locational-based Heuristics, which can derive good solutions in a short time. LBH is a type of cluster-first route-second method, and is an algorithm that clusters the customers into each vehicle and obtains a delivery route for each cluster. In the second stage, delivery destinations are clustered for each vehicle by solving an optimization problem called a Vehicle Location Problem (VLP) that is reduced to the Facility Location Problem (FLP). Some stores are selected as seed points and a virtual route (cluster) that goes back and forth between the depot and the seed points is created. Then, clustering is performed by inserting the remaining stores into one of the routes so as to satisfy the capacity constraint of each vehicle. By interpreting the roundtrip cost between the depot and the seed point is regarded as the location cost and the insertion cost as transportation cost, the problem of minimizing the sum of both costs is regarded as FLP. FLP is an NP-hard problem, but it can also be applied to large-scale problems because there is an efficient solution based on Lagrange relaxation. In the third stage, the delivery route for each cluster is determined by solving the Traveling Salesman Problem (TSP).

\subsection{Second stage: Vehicle Location Problem}

In the second stage, the delivery vehicle for each store is determined. Let $\hat{y}_{i t r}$ denote a binary variable that takes 1 if the delivery to the store $i$ in period $t$ by vehicle of type $r$ is selected as a seed point, and 0 otherwise. Let $c_{i r}^{S}$ denote the cost when the delivery to the store $i$ in period $t$ by vehicle of type $r$ is selected as a seed point. It is set as the round-trip cost between store $i$ and the depot, as follows.

$$
c_{i r}^{S}=c_{r}^{F}+c_{0 i r}^{V}+c_{i 0 r}^{V}
$$

Also, for non-seed store $j$, let $\hat{x}_{i j t r}$ denote a binary variable that takes 1 if store $j$ insert into store $i$ in period $t$, and 0 otherwise. Let $c_{i j t r}^{I}$ denote the cost of inserting the store $j$ into the seed point $i$ in cluster $C_{t r}$, and is set as follows,

$$
c_{i j t r}^{I}=c_{0 i r}^{V}+c_{i j r}^{V}-c_{0 j r}^{V}
$$




$$
\begin{aligned}
& \min . \quad \sum_{i \in V_{t r}^{\prime}} c_{i r}^{S} \hat{y}_{i t r}+\sum_{i \in V_{t r}^{\prime}} \sum_{j \in V_{t r}^{\prime}} c_{i j r}^{I} \hat{x}_{i j t r} \\
& \text { subject to } \\
& \sum_{j \in V_{t r}^{\prime}} \hat{x}_{i j t r}=1, \quad \forall i \in V_{t r}^{\prime} \\
& \sum_{j \in V^{\prime}} \hat{q}_{i t s r} \hat{x}_{i j t r} \leq Q_{k} \hat{y}_{i t r}, \quad \forall i \in V^{\prime}, \\
& \hat{x}_{i j t r} \leq \hat{y}_{i t r}, \quad \forall i \in V^{\prime}, \forall j \in V^{\prime} \\
& \hat{x}_{i j t r} \in\{0,1\}, \quad \forall i \in V_{t r}^{\prime}, \forall j \in V_{t r}^{\prime}, \forall s \in S, \\
& \hat{y}_{i t r} \in\{0,1\}, \quad \forall i \in N, \forall t \in T,
\end{aligned}
$$

The objective function (4a) shows the sum of the seed point installation cost and the store insertion cost, respectively. The constraint (4b) indicates that each store in the cluster $C_{t r}$ is assigned to one of the seed points. The constraint (4c) indicates the load limit for each vehicle. The constraint (4d) indicates that each store cannot be assigned to non-seed points. The constraint $(4 \mathrm{e})(4 \mathrm{f})$ indicates the binary condition of $\hat{x}_{i j t k}, \hat{y}_{i t k}$.

\subsection{Third stage: Traveling Salesman Problem}

By the second stage clustering, stores can be clustered into clusters by vehicle $k$ in period $t$, denoted as $C_{t k}$. Let $V_{t k}$ denote a set of stores contained in cluster $C_{t k}$. The routing decision for cluster $C_{t k}$ can be obtained by solving the problem (TSP $\left.P_{t k}\right)$ formulated as Eq. (5).

$$
\begin{aligned}
& \min . \quad \sum_{i \in V_{t k}} \sum_{j \in V_{t k}} c_{i j k}^{V} x_{i j t k} \\
& \sum_{i \in V_{t k}}^{\text {subject to }} x_{i j t k}=1 \quad \forall j \in V_{t k} \\
& \sum_{j \in V_{t k}} x_{i j t k}=1 \quad \forall i \in V_{t k} \\
& x_{i j t k}+x_{j i t k} \leq 2 \quad \forall \in V_{t k} \\
& \sum_{i, j \in X} x_{i j t k} \leq|X|-1 \\
& x_{i j t k} \in\{0,1\}, \quad \forall i, \forall j \in V_{t k}
\end{aligned}
$$

The objective function (5a) indicates the minimization of variable costs. The constraint $(5 b)(5 c)$ indicates that each node is visited once. The constraint $(5 \mathrm{~d})$ indicates the flow conservation of the route. The constraint $(5 \mathrm{e})$ indicates the sub tour elimination. The constraint (5f) indicates the binary condition of $x_{i j t k}$. The problem (5) is a Symmetric-TSP with $c_{i j k}^{V}=$ $c_{j i k}^{V}$, and an efficient exact method to a large-scale problem has been proposed. TSPLIB, which is a typical benchmark problem, reports the optimal solution for instances of $n>10^{5}$ or more.

\section{Numerical example}

This section shows application examples. In this study, we conducted numerical experiments based on actual data in the restaurant chain.

\subsection{Experimental setting and datasets}

The data set used in this experiment is as follows. For confidentiality reasons, demand, storage capacity, and sanctions limits have been normalized to $[0-1]$.

- $\quad$ Number of stores: $n=300$

- Stores where large vehicles can enter: $n_{S}=150$

- $\quad$ Demand: Table 3

- $\quad$ Store storage capacity: Table 4

- $\quad$ Fixed / variable / loading restrictions for vehicles: Table 5

The experimental environment is Intel (R) Core (TM) i7-8700T CPU 2.4GHz, 2.4GHz, 8.00GB memory. The optimization solver used was Gurobi Optimizer. The exact solution technique using Lazy Cuts (Applegate 2006) was applied to the TSP. 
Table 3

Demand Statistics

\begin{tabular}{|c|c|c|c|}
\hline Item & Day & Mean & Std. Dev. \\
\hline Dry $(s=1)$ & Weekday $(t=1, \cdots, 5)$ & 0.039 & 0.015 \\
\hline Dry $(s=1)$ & Weekend $(t=6,7)$ & 0.072 & 0.028 \\
\hline Chilled $(s=2)$ & Weekday $(t=1, \cdots, 5)$ & 0.015 & 0.006 \\
\hline Chilled $(s=2)$ & Weekend $(t=6,7)$ & 0.027 & 0.011 \\
\hline Frozen $(s=3)$ & Weekday $(t=1, \cdots, 5)$ & 0.033 & 0.013 \\
\hline Frozen $(s=3)$ & Weekend $(t=6,7)$ & 0.059 & 0.023 \\
\hline
\end{tabular}

Table 4

Capacity Statistics

\begin{tabular}{ccc}
\hline Item & Mean & Std. Dev. \\
\hline Dry $(s=1)$ & 0.26 & 0.12 \\
Chilled $(s=2)$ & 0.30 & 0.15 \\
Frozen $(s=3)$ & 0.52 & 0.19 \\
\hline
\end{tabular}

Table 5

Vehicle type parameters

\begin{tabular}{ccccc}
\hline$r$ & Size & Temp. Zone & Fixed Cost & Variable Cost \\
\hline 1 & Middle & Three Temp. & 90 & 0.59 \\
2 & Middle & Room Temp. & 83 & 0.54 \\
3 & Large & Three Temp. & 92 & 0.56 \\
4 & Large & Room Temp. & 85 & 0.60 \\
\hline
\end{tabular}

\subsection{Results}

As a benchmark, we compared with the following two-stage methods that are being used in the real-world operation. In the first stage, the delivery day and delivery amount are determined. The initial inventory on Monday is set as, the replenishment occurs right before the period when the inventory is negative up-to the inventory level again. In the second stage, the VRP is solved to get the optimal route. The vehicle type is selected by using a large three-temperature zone vehicle for large intrudable stores and a medium-sized three-temperature zone vehicle for other stores.

The experimental results are shown in Table 6. In addition, the breakdown of the number of vehicles used for each vehicle type is shown in table 7. Here, let be the number of vehicles used for vehicle type, and let denote the total number of vehicles used. From table 6, both the cost and the number of vehicles of the proposed technique are reduced. On the other hand, the number of deliveries for each store is increased. It is considered that this is because the comparison technique places orders for each store in a disorderly manner, whereas the proposed technique places orders for stores that are spatially close to each other on the same day as long as possible. Also, from table 7, in case \#0, all vehicle types are used, while in case \# 1 , by combining room-temperature vehicles, the number of deliveries can be reduced.

From this result, it is implied that even if the number of deliveries increases, the overall cost and number of vehicles can be reduced by using a room-temperature vehicle, which is cheaper than using a three-temperature vehicle to deliver items in each temperature zone to the same store all at once.

Table 6

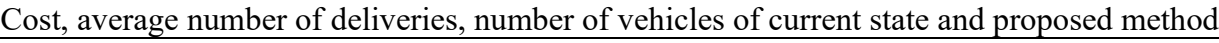

\begin{tabular}{cccccc}
\hline$\#$ & Model & $\begin{array}{c}\text { Fixed Cost } \\
(\$)\end{array}$ & $\begin{array}{c}\text { Variable Cost } \\
(\$)\end{array}$ & $\begin{array}{c}\text { Total Cost } \\
(\$)\end{array}$ & $\begin{array}{c}\text { Average Number of } \\
\text { Deliveries } \\
(\text { Times/week) }\end{array}$ \\
\hline$\# 0$ & Present & 4,720 & 4,807 & $\begin{array}{c}\text { Number of } \\
\text { Vehicles }\end{array}$ & 2.50 \\
\hline 1 & Proposed & 2,463 & 4,734 & 7,197 & 3.09 \\
\hline
\end{tabular}

Table 7

Breakdown of the number of vehicles used by

\begin{tabular}{cccccc}
\hline$\#$ & Model & $M$ & $M_{1}$ & $M_{2}$ & $M_{3}$ \\
\hline$\# 0$ & Present & 52 & 32 & 0 & 20 \\
$\# 1$ & Proposed & 29 & 5 & 18 & 0 \\
\hline
\end{tabular}

\subsection{Effect of using multiple vehicle types}

In order to clarify the effect of using multiple vehicle types together, experiments were conducted by changing the vehicle type used. Case \#2 is for medium-sized vehicles only $(R=\{1,2\})$, and case \#3 is for medium-sized three-temperature vehicles only $(R=\{1\})$. The results are shown in the Table 6 and Table 7 . From Table 8 , the more vehicle type options, the smaller the cost, average number of deliveries, and number of vehicles. In addition, from the Table 9 , in case \#1, by utilizing a large room-temperature vehicle, the number of medium-sized three-temperature vehicles and medium-sized room-temperature vehicles can be reduced, compared with cases \#2 and \#3. These results indicate the effectiveness of 
optimization of inventory routing decisions with multiple vehicle types with different characteristics is validated.

Table 8

Cost Average Delivery Frequency, Number of Vehicles when the available vehicle types are changed

\begin{tabular}{|c|c|c|c|c|c|c|}
\hline \# & Model & Fixed Cost (\$) & Variable Cost (\$) & $\begin{array}{l}\text { Total Cost } \\
\text { (\$) }\end{array}$ & $\begin{array}{c}\text { Average Number of } \\
\text { Deliveries } \\
\text { (Times/week) }\end{array}$ & $\begin{array}{c}\text { Number of } \\
\text { Vehicles }\end{array}$ \\
\hline$\# 1$ & $\begin{array}{c}\text { All Vehicle Type } \\
(R=\{1,2,3,4\})\end{array}$ & 246300 & 473413 & 719713 & 3.09 & 29 \\
\hline \#2 & $\begin{array}{l}\text { Middle Sized Vehicle } \\
\quad(R=\{1,2\})\end{array}$ & 305800 & 551705 & 857505 & 3.36 & 36 \\
\hline$\# 3$ & $\begin{array}{c}\text { Middle Sized Three-temp. } \\
\text { Vehicle } \\
(R=\{1\})\end{array}$ & 351000 & 562921 & 913921 & 3.47 & 39 \\
\hline
\end{tabular}

Table 9

Breakdown of the number of vehicles by type when the available vehicle types are changed

\begin{tabular}{|c|c|c|c|c|c|c|}
\hline$\#$ & Model & $M$ & $M_{1}$ & $M_{2}$ & $M_{3}$ & $M_{4}$ \\
\hline$\# 1$ & $\begin{array}{c}\text { All Vehicle Type } \\
(R=\{1,2,3,4\})\end{array}$ & 29 & 5 & 18 & 0 & 6 \\
\hline$\# 2$ & $\begin{array}{l}\text { Middle Sized Vehicle } \\
\quad(R=\{1,2\})\end{array}$ & 36 & 10 & 26 & 0 & 0 \\
\hline$\# 3$ & $\begin{array}{c}\text { Middle Sized Three-temp. } \\
\text { Vehicle } \\
(R=\{1\})\end{array}$ & 39 & 39 & 0 & 0 & 0 \\
\hline
\end{tabular}

\subsection{Effect of Site dependency}

In order to see the effect of site dependency, we conducted an experiment by changing the number of stores where large vehicles could visit. $n_{s}$ is the number of stores that large vehicles can visit. We changed this parameter as $n_{s}=$ $0,75,150,225,300$. The results are shown in the Table 10 and Table 11. It can be confirmed that the total number of vehicles used is decreasing as the number of stores where large vehicles can visit increases. It can also be seen that the types of vehicles used are also changing. In cases \#1 and \#5, medium-sized vehicles occupy the majority, and large three-temperature zone vehicles are not used. On the other hand, in case \#6, all vehicle types are used in a well-balanced manner. Furthermore, in case \#7, medium-sized three-temperature vehicles are not used, and large vehicles account for most deliveries.

From this result, the optimum vehicle type depends on the ratio of stores where large vehicles can visit. It is implied that a significant cost reduction can be achieved by selecting an appropriate vehicle type. It implies that the necessity and effectiveness of the optimization approach with multi-vehicle options could be verified.

Table 10

Cost, average number of deliveries, number of vehicles when changing the site dependency

\begin{tabular}{|c|c|c|c|c|c|c|}
\hline$\#$ & Model & Fixed Cost (\$) & Variable Cost (\$) & $\begin{array}{c}\text { Total Cost } \\
\text { (\$) }\end{array}$ & $\begin{array}{c}\text { Average Number of } \\
\text { Deliveries } \\
\text { (Times/week) }\end{array}$ & $\begin{array}{c}\text { Number of } \\
\text { Vehicles }\end{array}$ \\
\hline$\# 4$ & 0 & 304400 & 560531 & 864931 & 3.68 & 40 \\
\hline$\# 5$ & 75 & 305200 & 522779 & 827979 & 3.28 & 34 \\
\hline$(\# 1)$ & 150 & 246300 & 473413 & 719713 & 3.09 & 29 \\
\hline$\# 6$ & 225 & 239600 & 426242 & 665842 & 3.24 & 28 \\
\hline$\# 7$ & 300 & 205200 & 439784 & 644984 & 2.84 & 22 \\
\hline
\end{tabular}

Table 11

Breakdown of the number of vehicles when changing the site dependency

\begin{tabular}{ccccccc}
\hline$\#$ & Model & $M$ & $M_{1}$ & $M_{2}$ & $M_{3}$ \\
\hline$\# 4$ & 0 & 40 & 8 & 32 & 0 \\
$\# 5$ & 75 & 34 & 9 & 22 & 0 \\
$(\# 1)$ & 150 & 29 & 5 & 18 & 0 \\
$\# 6$ & 225 & 28 & 7 & 9 & 3 \\
$\# 7$ & 300 & 22 & 0 & 4 & 9 \\
\hline
\end{tabular}

\section{Conclusions}

By collaborating with the logistics company and the customer to make a decision that integrates the customer's inventory management and the delivery plan of the logistics company, a dramatic improvement in delivery efficiency can be expected. Against this background, this study conducted a study of IRP considering multiple temperature zones and multiple vehicle types for restaurant chains. The problem was formulated as a Multi-Product Multi-Vehicle IRP. 
Since MMIRP is a difficult problem to solve, it was difficult to apply it to large-scale problems. Therefore, in this study, we proposed three-step Math Heuristics based on the cluster destination and post-root method. In the first stage, by solving the Multi-item Multi-vehicle Inventory Problem (MMP), the delivery day, delivery vehicle type, delivery day, delivery vehicle type, etc. for each store so that the vehicle fixed cost is minimized while satisfying the upper and lower limit constraints of inventory. Determine the delivery amount. From the output of this MMIP, a delivery destination cluster can be created for each delivery day and delivery vehicle type. In the second stage, the vehicle location problem (VIP) is solved to determine the delivery vehicle for each store so that the approximate delivery variable cost is minimized. The output of this VLP creates a delivery destination cluster for each delivery vehicle. In the third stage, the Traveling Salesman Problem (TSP) is used to determine the delivery route.

In the numerical experiment, verification was performed using actual data. It was concluded that large-sized / threetemperature vehicles would be used for large stores, and medium-sized / three-temperature vehicles and medium-sized / room-temperature vehicles would be used together for medium-sized stores. We got the need for IRP and useful suggestions for solutions. In addition, a sensitivity analysis was conducted to consider the effects of changes in vehicle type, total number of vehicles, and demand on the results.

As a future issue, application to further large-scale problems can be considered. In particular, the proposed MMIP could be solved, but it becomes difficult to solve it as the number of stores, items, and vehicle types increases. As one method, a method using Lagrange relaxation can be considered. By relaxing each constraint, MMIP can be broken down into storeby-store decisions. Moreover, the decomposed problem can be solved efficiently by using the dynamic programming method.

\section{Acknowledgement}

This work was supported by JSPS KAKENHI Grant Number 19K04894.

\section{References}

Aksen, D., Kaya, O., Salman, F. S., \& Akça, Y. (2012). Selective and periodic inventory routing problem for waste vegetable oil collection. Optimization Letters, 6(6), 1063-1080.

Alkawaleet, N., Hsieh, Y. F., \& Wang, Y. (2014). Inventory routing problem with CO 2 emissions consideration. In Logistics operations, supply chain management and sustainability (pp. 611-619). Springer, Cham.

Applegate, D. L., Bixby, R. E., Chvatal, V., \& Cook, W. J. (2006). The traveling salesman problem: a computational study. Princeton university press.

Archetti, C., Bertazzi, L., Laporte, G., \& Speranza, M. G. (2007). A branch-and-cut algorithm for a vendor-managed inventory-routing problem. Transportation science, 41(3), 382-391.

Avella, P., Boccia, M., \& Wolsey, L. A. (2018). Single-period cutting planes for inventory routing problems. Transportation Science, 52(3), 497-508.

Baldacci, R., Battarra, M., \& Vigo, D. (2008). Routing a heterogeneous fleet of vehicles. In The vehicle routing problem: latest advances and new challenges (pp. 3-27). Springer, Boston, MA.

Bell, W. J., Dalberto, L. M., Fisher, M. L., Greenfield, A. J., Jaikumar, R., Kedia, P., ... \& Prutzman, P. J. (1983). Improving the distribution of industrial gases with an on-line computerized routing and scheduling optimizer. Interfaces, 13(6), 423.

Benoist, T., Gardi, F., Jeanjean, A., \& Estellon, B. (2011). Randomized local search for real-life inventory routing. Transportation Science, 45(3), 381-398.

Bertazzi, L., Speranza, M. G., \& Ukovich, W. (1997). Minimization of logistic costs with given frequencies. Transportation Research Part B: Methodological, 31(4), 327-340.

Chao, I. M., Golden, B., \& Wasil, E. (1999). A computational study of a new heuristic for the site-dependent vehicle routing problem. INFOR: Information Systems and Operational Research, 37(3), 319-336.

Christiansen, M., Fagerholt, K., Flatberg, T., Haugen, Ø., Kloster, O., \& Lund, E. H. (2011). Maritime inventory routing with multiple products: A case study from the cement industry. European Journal of Operational Research, 208(1), 8694.

Coelho, L. C., Cordeau, J. F., \& Laporte, G. (2014). Thirty years of inventory routing. Transportation Science, 48(1), 1-19.

Coelho, L. C., \& Laporte, G. (2013). A branch-and-cut algorithm for the multi-product multi-vehicle inventory-routing problem. International Journal of Production Research, 51(23-24), 7156-7169.

Cordeau, J. F., Laganà, D., Musmanno, R., \& Vocaturo, F. (2015). A decomposition-based heuristic for the multiple-product inventory-routing problem. Computers \& Operations Research, 55, 153-166.

Cousineau-Ouimet, K. (2002, November). A tabu search heuristic for the inventory routing problem. In Proceedings of 37th Annual ORSNZ Conference.

Dauzère-Pérès, S., Nordli, A., Olstad, A., Haugen, K., Koester, U., Per Olav, M., ... \& Reistad, A. (2007). Omya Hustadmarmor optimizes its supply chain for delivering calcium carbonate slurry to European paper manufacturers. Interfaces, 37(1), 39-51.

Desaulniers, G., Rakke, J. G., \& Coelho, L. C. (2016). A branch-price-and-cut algorithm for the inventory-routing problem. 
Transportation Science, 50(3), 1060-1076.

Ercan, S., \& Cinar, D. (2017). A Multiproduct Multi-vehicle Inventory Routing Problem with Uncertainty. In Sustainable Logistics and Transportation (pp. 181-198). Springer, Cham.

Gaur, V., \& Fisher, M. L. (2004). A periodic inventory routing problem at a supermarket chain. Operations Research, 52(6), 813-822.

Guerrero, W. J., Prodhon, C., Velasco, N., \& Amaya, C. A. (2013). Hybrid heuristic for the inventory location-routing problem with deterministic demand. International Journal of Production Economics, 146(1), 359-370.

Hemmelmayr, V., Doerner, K. F., Hartl, R. F., \& Savelsbergh, M. W. (2009). Delivery strategies for blood products supplies. OR spectrum, 31(4), 707-725.

Kleywegt, A. J., Nori, V. S., \& Savelsbergh, M. W. (2004). Dynamic programming approximations for a stochastic inventory routing problem. Transportation Science, 38(1), 42-70.

Koç, Ç., Bektaş, T., Jabali, O., \& Laporte, G. (2016). Thirty years of heterogeneous vehicle routing. European Journal of Operational Research, 249(1), 1-21.

Liu, S. C., \& Lee, W. T. (2011). A heuristic method for the inventory routing problem with time windows. Expert Systems with Applications, 38(10), 13223-13231.

Liu, S. C., \& Chen, A. Z. (2012). Variable neighborhood search for the inventory routing and scheduling problem in a supply chain. Expert Systems with Applications, 39(4), 4149-4159.

Lmariouh, J., Coelho, L. C., Elhachemi, N., Laporte, G., Jamali, A., \& Bouami, D. (2017). Solving a vendor-managed inventory routing problem arising in the distribution of bottled water in Morocco. European Journal of Industrial Engineering, 11(2), 168-184.

Mirzapour Al-e-hashem, S. M. J., \& Rekik, Y. (2014). Multi-product multi-period Inventory Routing Problem with a transshipment option: A green approach. International Journal of Production Economics, 157, 80-88.

Mjirda, A., Jarboui, B., Macedo, R., Hanafi, S., \& Mladenović, N. (2014). A two phase variable neighborhood search for the multi-product inventory routing problem. Computers \& Operations Research, 52, 291-299.

Mjirda, A., Jarboui, B., Macedo, R., \& Hanafi, S. (2012). A variable neighborhood search for the multi-product inventory routing problem. Electronic Notes in Discrete Mathematics, 39, 91-98.

Moin, N. H., Salhi, S., \& Aziz, N. A. B. (2011). An efficient hybrid genetic algorithm for the multi-product multi-period inventory routing problem. International Journal of Production Economics, 133(1), 334-343.

Nag, B., Golden, B. L., \& Assad, A. (1988). Vehicle routing with site dependencies. Vehicle routing: Methods and studies, 149-159.

Oppen, J., Løkketangen, A., \& Desrosiers, J. (2010). Solving a rich vehicle routing and inventory problem using column generation. Computers \& Operations Research, 37(7), 1308-1317.

Park, Y. B., Yoo, J. S., \& Park, H. S. (2016). A genetic algorithm for the vendor-managed inventory routing problem with lost sales. Expert Systems with Applications, 53, 149-159.

Popović, D., Vidović, M., \& Radivojević, G. (2012). Variable neighborhood search heuristic for the inventory routing problem in fuel delivery. Expert Systems with Applications, 39(18), 13390-13398.

Rahimi, M., Baboli, A., \& Rekik, Y. (2017). Inventory routing problem for perishable products by considering customer satisfaction and green criteria. In Dynamics in Logistics (pp. 445-455). Springer, Cham.

Ronen, D. (2002). Marine inventory routing: Shipments planning. Journal of the Operational Research Society, 53(1), 108114.

Song, J. H., \& Furman, K. C. (2013). A maritime inventory routing problem: Practical approach. Computers \& Operations Research, 40(3), 657-665.

Speranza, M. G., \& Ukovich, W. (1994). Minimizing transportation and inventory costs for several products on a single link. Operations Research, 42(5), 879-894.

Speranza, M. G., \& Ukovich, W. A. L. T. E. R. (1996). An algorithm for optimal shipments with given frequencies. Naval Research Logistics (NRL), 43(5), 655-671.

Su, Z., Lu, Z., Wang, Z., Qi, Y., \& Benlic, U. (2020). A matheuristic algorithm for the inventory routing problem. Transportation Science, 54(2), 330-354.

Treitl, S., Nolz, P. C., \& Jammernegg, W. (2014). Incorporating environmental aspects in an inventory routing problem. A case study from the petrochemical industry. Flexible Services and Manufacturing Journal, 26(1-2), 143-169.

Wang, Q., Ji, Q., \& Chiu, C. H. (2014). Optimal routing for heterogeneous fixed fleets of multicompartment vehicles. Mathematical Problems in Engineering, 2014.

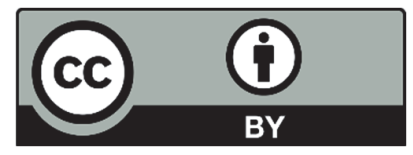

(C) 2021 by the authors; licensee Growing Science, Canada. This is an open access article distributed under the terms and conditions of the Creative Commons Attribution (CC-BY) license (http://creativecommons.org/licenses/by/4.0/). 